\title{
THE DESIGN OF A CATHODE TO OPERATE IN AN OXYGEN-RICH ENVIRONMENT
}

\author{
Colleen M. Marrese and \\ Alec D. Gallimore \\ Plasmadynamic \& Electric Propulsion Lab. \\ The University of Michigan \\ Dept. of Aerospace Engineering \\ FXB Building, 1320 Beal \\ Ann Arbor, MI 48109-2118 \\ (313) 764-4199
}

\author{
William A. Mackie and \\ David E. Evans \\ Linfield Research Institute \\ 900 Baker St. \\ McMinnville, OR 97128-6894 \\ billm@linfield.edu \\ (503) 434-2432
}

\begin{abstract}
The primary problem with Hall plasma accelerator operation on oxygen is poor cathode performance and short lifetime. The primary problem with micro Hall thrusters is the absence of a stable low power cathode. Cathodes traditionally used for both applications employ thermionic emitters which are not efficient and which are easily oxidized in an oxygen-rich environment. The field emitter cathode presented in this report has the potential of filling both vacancies since it does not require a high-power heater and can be scaled down with the size of the thruster. The advantages to using $\mathrm{Hf}$ and $\mathrm{HfC}$ as emitting materials are low work functions and high resistance to oxygen poisoning. Preliminary investigations proved that $\mathrm{HfC}$ emitters can operate in $7.6 \mathrm{~m}$ Torr oxygen pressure environments. The initial cathode design employs an electrostatic lens that also acts as an ion filter to prevent thruster ions from bombarding the field emitters while decelerating the electron beam and keeping it focused to ensure efficient performance. Electron trajectories through the cathode and ion filtering capabilities are presented in this report as predicted by the charged particle code, MAGIC.
\end{abstract}

\section{INTRODUCTION}

Since the lifetime of most spacecraft is limited by the amount of on-board propellant, it would be economically advantageous to develop a propulsion system that can utilize in-situ propellant. With $50 \%$ of the surface of the moon composed of oxygen, oxygen-fueled Hall thrusters could be used to propel trans-lunar cargo vessels to the moon and beyond. Multi-kilowatt propulsion systems or arrays of low-power Hall thrusters could be used on such spacecraft.

Hall thrusters capable of running on oxygen have many applications in the material processing field as well. Oxygen plasma sources are commonly used for oxide film deposition, ion beam assisted deposition, reactive ion beam etching, substrate cleaning in the micro-processing industry, and material property enhancement. The advantages of using ion beam assisted processing over chemical processing are better surface adhesion of the coatings, better coverage of thin films, and higher density films. Therefore, coatings are thinner and stronger, and optical films have higher optical indicies with decreased permeability to water. The plasma sources typically employed are radio frequency (RF) and electron cyclotron resonance (ECR) types, as well as Kauffman sources (ion thrusters). Since ion current densities in ion engines are spacecharge limited, they are typically lower than those achieved by Hall thrusters. When Hall thrusters replace ion engines for the same applications, processing times can be significantly reduced. By using an array of low-power Hall thrusters, increased current densities with comparable plume uniformities relative to ion thrusters can be achieved. The cathode presents the primary difficulty in the operation of Hall thrusters on $\mathrm{O}_{2}$.

Cathodes that are typically used on Hall thrusters employ a thermionic emitter that require a high power heater for ignition and can be poisoned easily in the presence of oxygen. To increase lifetime, inert gases are typically used in the cathode. While some applications can tolerate a two propellant scheme, it complicates the propellant feed system and introduces impurities into the discharge that may not be desired for some material processing applications. An additional problem with the separate propellant required by the cathode is that it can become a significant percent of the total propellant flow rate through small propulsion systems. Therefore, it can be a significant source of thruster inefficiency. Another source of inefficiency is the high-power heater required for ignition. These cathodes have not been successfully scaled down with thruster size. Therefore, using currently existing cathodes is inefficient since cathode power consumption can become $50 \%$ of the total propulsion system power (Manzella et al. 1996). The recent drive to develop small Hall thrusters for micro satellites (Manzella et al. 1996 and Khayms et al. 1996) necessitates the development of a cathode that can operate at approximately $10 \%$ of the total thruster power of $50-450 \mathrm{~W}$. 
The cathode described in this report employs a field emitter array (FEA) that can be scaled down to different power levels by changing the number of emitters in the array. The two distinct advantages it offers over thermionic cathodes are the absence of a high power heater and propellant. Therefore, it is a promising candidate to replace thermionic cathodes for Hall thrusters that use oxygen or more typical propellants such as xenon or krypton.

This report describes field emitter arrays and typical performances, presents preliminary results from experiments testing single field emitters in oxygen-rich environments, the cathode configuration, and the predicted performance of the cathode design run in one of the three possible configurations using the PIC code, MAGIC.

\section{FIELD EMITTER ARRAYS}

In this section of the report a background on field emitter technology is given to motivate the current application, the performance requirements are presented for a FEC to operate in an oxygen rich environment, and the state of the research on the optimal field emitter material for this cathode is summarized.

Micro-fabricated FEA cathodes are being actively developed around the world for use in vacuum devices, such as flat panel displays, coherent radiation sources, and ultra fast logic circuits because of their low turn-on voltages, high current densities, and stable operation in a pulsed mode (Mackie et al. 1992). Typical FEAs employ molybdenum or silicon tips fabricated by etching techniques. The configuration of a single array element is shown in Figure 1. Emitters have a radius of curvature down to tens of angstroms (Mackie et al. 1996) and can be fabricated in arrays with packing densities on the order of $10^{6}$ tips $/ \mathrm{cm}^{2}$ (Mackie et al. 1995). Generated surface electric fields must be on the order of $10^{9} \mathrm{~V} / \mathrm{m}$ for good emission. Field emission currents per tip are typically in the few $\mu \mathrm{A}$ range for array elements but can be as high as $48 \mathrm{~mA}$ for single tip configurations if transition metal carbides (TMCs) are run in a pulsed mode (Mackie et al. 1993). Stable currents of $0.5 \mathrm{~mA}$ with lifetimes greater than $2400 \mathrm{hr}$. have been reported for carbide emitters (Mackie et al. 1993). Use of FEAs in lowpressure discharges has been discouraged in the past because the delicate tips easily degraded in shape, and therefore performance, under the high-energy ion bombardment induced by large gate electrode (VG) voltages. However, recent advances in micro-fabrication techniques and field emitter materials have resulted in lower gate voltages for the required emission currents (Mackie 1993), thereby reducing emitter cone sputter rates to improve performance.

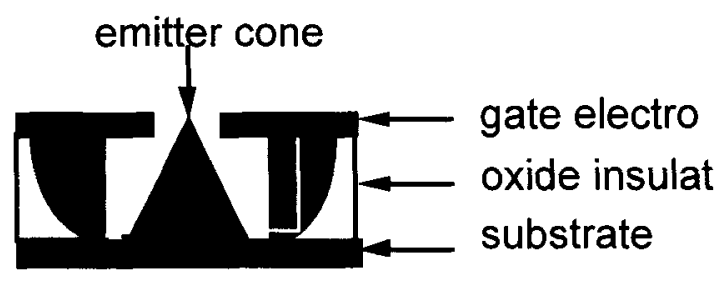

FIGURE 1. Schematic of a Single Element of the Field Emitter Arrays.

The performance requirements of the FEAs for this cathode include emitter currents in the $\mu \mathrm{A}$ range at 10-70 V gate voltages with high efficiency and high tip resistance to ion sputtering and oxygen poisoning. Low gate voltages are required to minimize the energy of ions bombarding the emitters and maintain electron energies $\sim 20 \mathrm{eV}$, thereby ensuring efficient ion beam neutralization and propellant ionization. Gate voltages above $20 \mathrm{~V}$ are tolerable when electrons are decelerated to $\sim 20 \mathrm{eV}$ before exiting the cathode. However, the optimal configuration would not require beam deceleration. Low leakage current of the FEA through the gate electrode is required to ensure efficient cathode operation. It is typical for the ratio of emitted current to gate current to be as high as 1000 . Therefore, the cathode power consumption due to this current will be $\sim .1 \mathrm{~mW}$. Tip resistance to ion sputtering and oxygen poisoning depends on emitter material. It is important to employ a material that will not be insulated by oxygen, not easily sputtered by ion bombardment, and has a low work function.

Group IVB transition metals and TMCs are currently under investigation to improve on emitter performance of molybdenum and silicon tips that are typically employed. Their advantages include superior hardness, chemical inertness, and work functions. These metals have an electropositive nature that reduces the work function of the emitters (Schwoebel et al. 1995). The most promising materials are $\mathrm{Hf}$ and $\mathrm{HfC}$ because of their high resistance to oxygen poisoning. Although 
$\mathrm{HfO}_{2}$ is readily formed during exposure of $\mathrm{Hf}$ and $\mathrm{HfC}$ to oxygen, it has a work function that is lower than both Mo and Si. Average work functions of Mo and Si are 4.6 and $4.85 \mathrm{eV}$ (Lide 1992), respectively, and work functions measured for Hf, $\mathrm{HfC}$, and $\mathrm{HfO}_{2}$ are 3.53 (Djuba et al. 1991) and 3.2 (Mackie et al. 1993), and $3.6 \mathrm{eV}$ (Djuba et al. 1991) respectively. The value of the work function given for $\mathrm{HfC}$ corresponds to $\mathrm{HfC}_{0.88}\{210\}$, however, it is believed that the work function of the $\{310\}$ crystal plane will be lower than the $\{210\}$ plane. Because it is possible to fabricate the emitters with the tip centered on specific crystal planes, the emitter work function can be controlled. The work function of the emitters also depends on material stoichiometry (Price et al. 1993). The work function of $\mathrm{HfC}$ was given for a carbon to metal ratio of 0.88 . Since the material stoichiometry has not yet been optimized, it is possible that $\mathrm{HfC}$ can be fabricated with lower work functions than $3.2 \mathrm{eV}$. The emission current dependence on the work function of the emitter and gate electrode voltage is described by a simplified version (Schwoebel et al. 1995) of the Fowler-Nordheim (FN) relationship (eq.1)

$$
\frac{1}{V^{2}}=\alpha \beta^{2} \exp \left(\frac{-b \phi^{\frac{3}{2}}}{\beta V}\right) \text {, }
$$

where $I$ is the electron current, $V$ is the applied voltage, $b$ is a constant, $a=a(\phi), \phi$ is the average work function of the surface, and $\beta$ is a proportionality factor between applied voltage and the magnitude of electric field at the emitting surface. Since the work functions of $\mathrm{Hf}$ and $\mathrm{HfC}$ are lower than $\mathrm{Mo}$ and $\mathrm{Si}$, lower voltages are required for the same emission current which reduces the energy of the ions bombarding the emitters. In addition to performing better because of lower work functions, it has been shown that short-term exposure to oxygen actually enhances the emission behavior of the carbides because it lowers the work function of the emitter (Mackie et al. 1994). Since the emitters had not been tested previously in $\sim 0.1 \mathrm{mTorr}$ oxygen environments that are required for this cathode, some preliminary investigations were conducted with $\mathrm{HfC}$ emitter tips.

\section{OPERATION OF HFC FIELD EMITTER TIPS IN OXYGEN-RICH ENVIRONMENTS}

To determine if $\mathrm{HfC}$ emitters will continue to operate in an oxygen rich environment several experiments were conducted at the Linfield Research Institute. Data were obtained in a preliminary investigation to ensure that the emitters could operate in an oxygen pressure environment that exceeds $10^{-4}$ Torr. Emitter tips centered on different crystal planes with varying geometries were operated in oxygen pressure environments ranging from $10^{-10}$ Torr to $7.6 \mathrm{mTorr}$. Emission patterns on the FE tips were observed on the phosphorus coated anode. In a configuration where the gap between the anode and emitter was $2 \mathrm{~cm}$, several kilovolts were required for emission. The emitters continued to operate at $7.6 \mathrm{mTorr}$ with $10 \mathrm{nA}$ of current. Currents as high as $2.6 \mu \mathrm{A}$ were measured at $1.9 \times 10^{-6}$ Torr $(9000 \mathrm{~V})$ and $0.32 \mu \mathrm{A}$ at $10^{-4}$ Torr $(4900 \mathrm{~V})$.

Some of the trends observed in the experiments are shown in Figure 2.. Typically, the emission pattern from the tips during pressure ramps only changed in intensity, however, in one experiment emission from a second set of crystal planes was enhanced significantly around $1.1 \times 10^{-6}$ Torr $(6000 \mathrm{~V})$. When emitter tips become contaminated the emission pattern is not representative of specific crystal planes, instead, electron emission is uniformly distributed over the tip. One of the most promising results of the experiments was that the tip emission patterns were not destroyed during operation in oxygen-rich environments. Another interesting result was the current increase with time when discharge voltage and tank pressure were held constant. This phenomenon is not uncommon in field emitter operation and has been explained by Mousa et al. (Mousa et al. 1993) as a reduction of work function possibly due to deadsorption from emitter surface of electronegative species like oxygen. The tips were also run in argon rich environments with similar results, showing that the field emitter arrays have the potential of being used with propulsion systems that use other propellants.

Since the environments tested are much more hostile than what the emitters will experience during operation with Hall thrusters, it is believed that they will operate much better in the true thruster environments. Pressures at the emitter tips should be on the order of $10^{-4}$ Torr with gate voltages less than $100 \mathrm{~V}$. Therefore, fewer ions will be bombarding the tips with energies as much as two orders of magnitude lower than tested. 


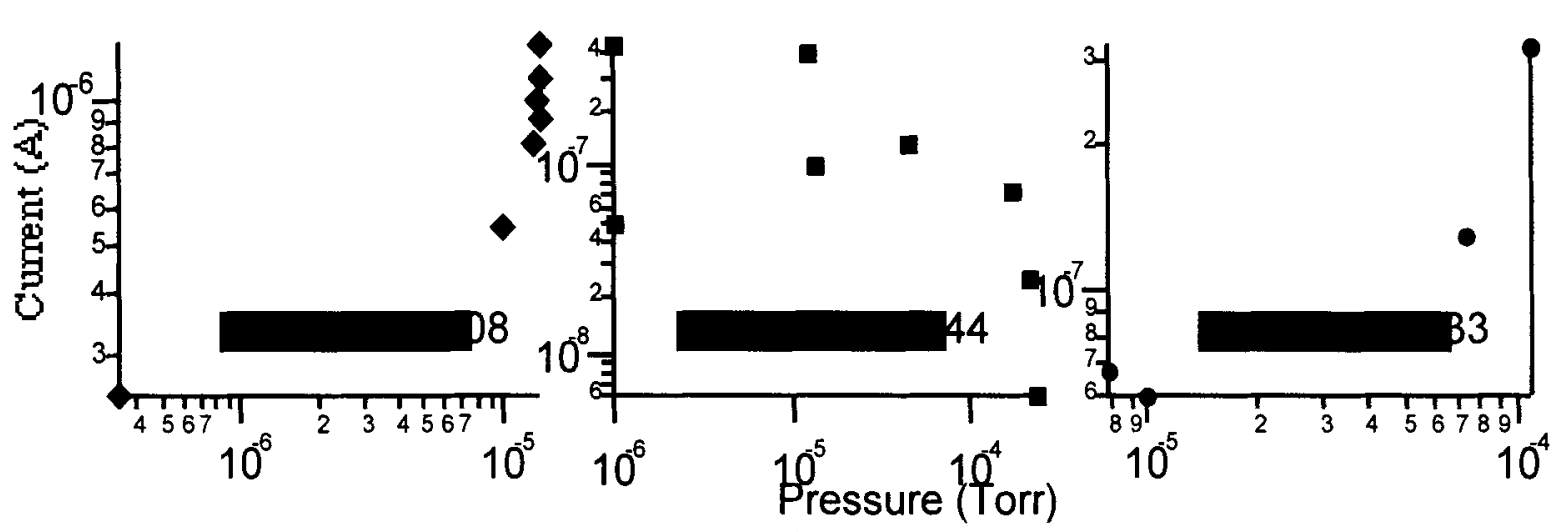

FIGURE 2. Emitter Currents Measured over Time Periods, $\Delta$ t, vs. Oxygen Pressure at the Specified Voltages.

\section{THE CATHODE}

In this section of the report one basic cathode design is presented that could be operated in three different configurations. In the first and simplest configuration, the cathode operates with a $20 \mathrm{~V}$ gate voltage and does not require a heater or a decelerating electrostatic lens. If FEAs are not capable of operating with $\sim 20 \mathrm{~V}$ gate voltages at the time of fabrication, then a second cathode configuration will employ the heater shown to thermally enhance electron emission from the tip to attain the required emission currents. The third cathode configuration does not employ a heater. It operates with gate voltages higher than $20 \mathrm{~V}$ to attain the required emission and decelerates the electron beam using the electrode configuration shown in Figure 3. This report focuses on the third cathode configuration.

In the third cathode configuration, electrodes $\mathrm{V} 1, \mathrm{~V} 2$, and $\mathrm{V} 3$ filter the ions and decelerate and focus the electron beam. Ions entering the cathode with kinetic energy below $65 \mathrm{eV}$ will be retarded by the electric field between V2 and V3. V1, V2, and V3 create an electric field to focus the beam through the high voltage grid V2. V3 also shields the ion retarding grid from the electrons in the thruster discharge. Electrode potentials are given in Table 1.

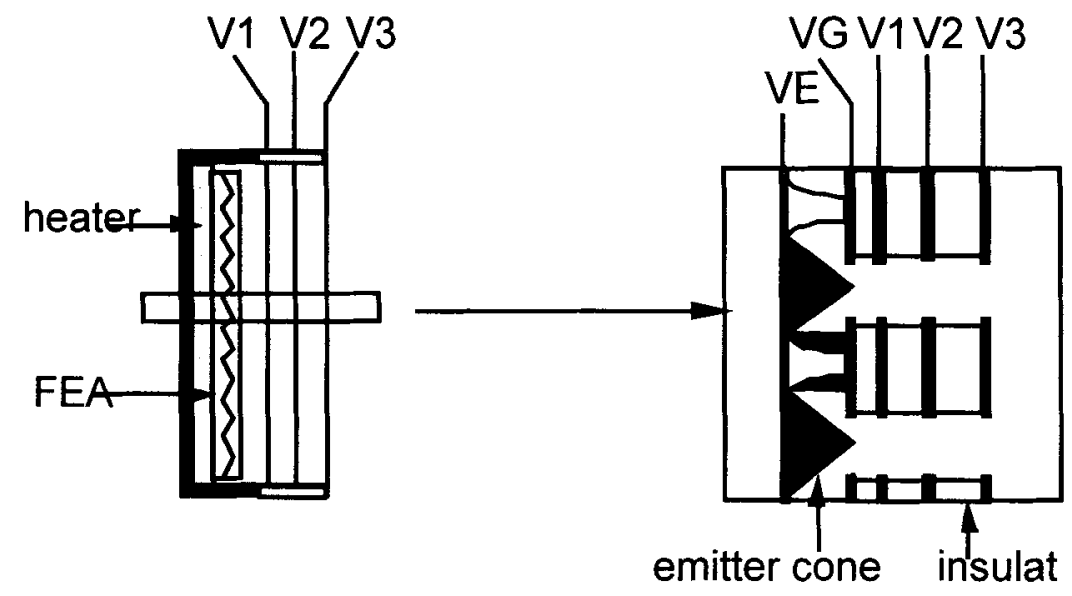

FIGURE 3. FEA Cathode Configuration.

\section{PERFORMANCE BY MAGIC}

Cathode design development was aided by the particle-in-cell (PIC) code MAGIC. This code, developed by Mission Research Corporation, provides interaction between space charge and electromagnetic fields. It is a two-dimensional, finitedifference, time-domain code that simulates plasma physics processes. Electromagnetic fields are determined using the full set of Maxwell's time-dependent equations. Current and charge densities for Maxwell's equations are obtained by solving the complete Lorenz force equations.

MAGIC was used to evaluate the third cathode configuration proposed The simulation was run for a beamlet generated by one emitter with a series of single aperture electrodes. Only half of this configuration is shown in Figures 4 and 5 because of 
azimuthal symmetry. The potentials applied to the electrodes with respect to ground in the simulation and the thicknesses of the electrodes, $t$, and insulators, $d$, are included in Table 1. Figure 4 shows equipotentials demonstrating the focusing capability of the cathode when it operated in a decelerating mode. It also shows that a $100 \mathrm{~V}$ electrode potential was required to filter out $65 \mathrm{~V}$ ions due to the electrode spacing required by the lens. Figure 5 shows the electron trajectories with an anode plate at $15 \mathrm{~V}$ to simulate the plasma. The design objective to decelerate and focus the beam was achieved.

TABLE 1. Electrode Voltages (V), Electrode Spacings ( $\mu \mathrm{m})$, and Electrode Thicknesses $(\mu \mathrm{m})$ Employed in the MAGIC Simulation.

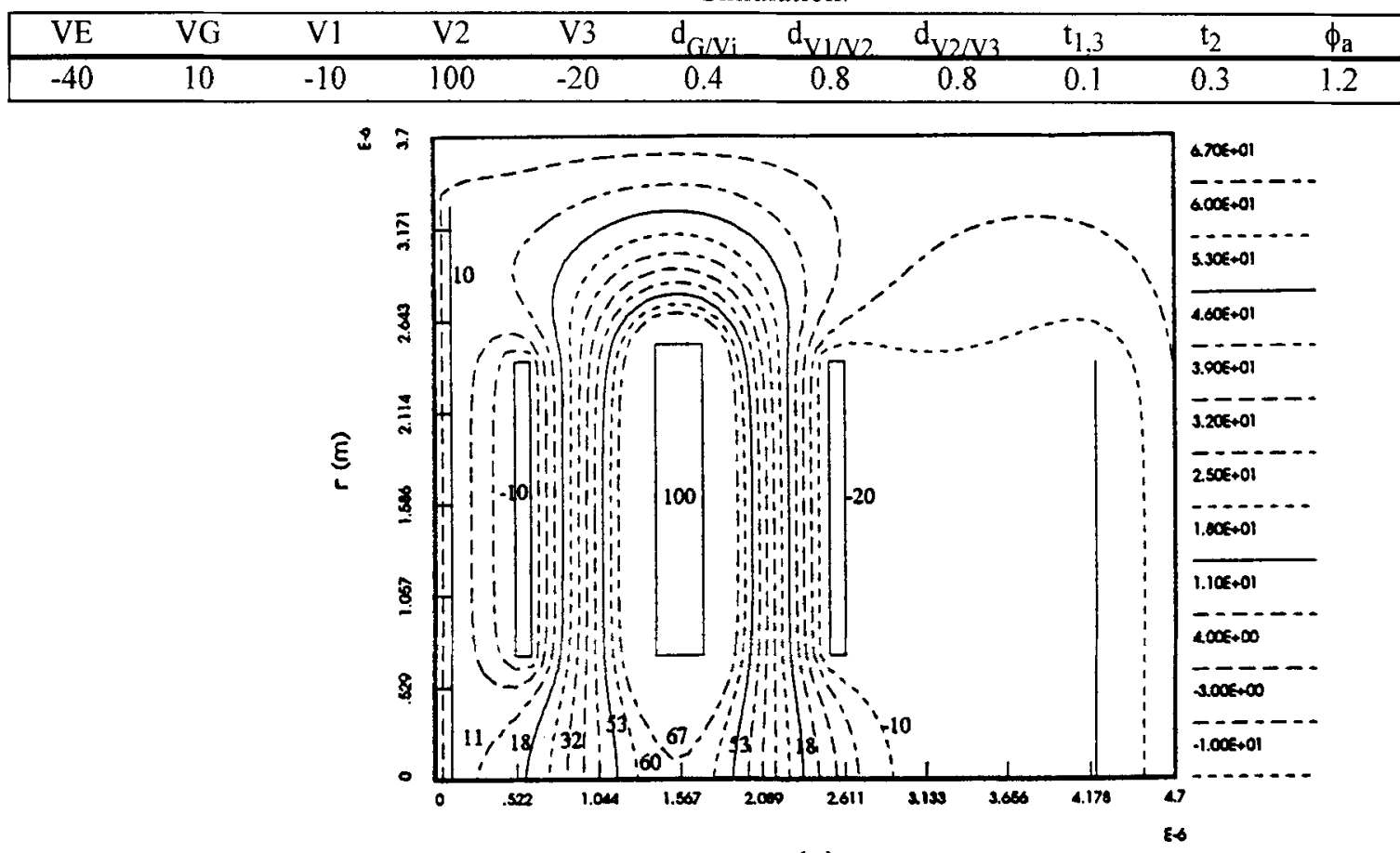

FIGURE 4. Equipotentials for the Electrostatic Lens in the FEC.

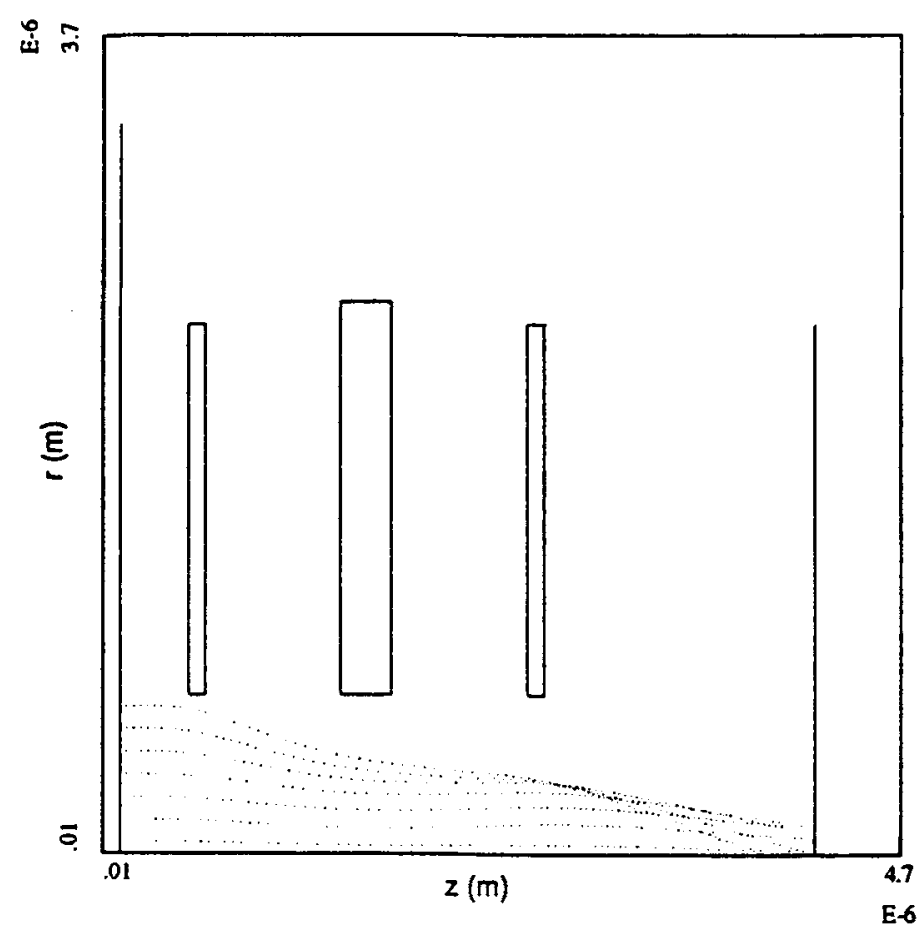

FIGURE 5. Electron Trajectories from a Single Field Emitter Through the Electrostatic Lens. 


\section{CONCLUSIONS AND FUTURE WORK}

This paper reports on design concepts for a cathode that could operate in an oxygen-rich environment. The electrode configuration in the decelerating cathode design presented was evaluated by MAGIC to ensure that the electron beam would escape through the lens electrodes while it was being decelerated down to the required energies. HfC field emitters were tested in a high oxygen pressure environment to prove that they can tolerate hostile environments and are, therefore, promising candidates for the electron emitting material in the oxygen cathode. The future work includes fabrication and testing of the cathode design presented. Specific $\mathrm{HfC}$ and $\mathrm{Hf}$ field emitter arrays must be chosen that are capable of emitting 1-5 A with lifetimes in excess of $2000 \mathrm{hr}$. Gate electrode material must be chosen to provide low oxidation rates and low sputter rates. The configuration of the gate electrode must be optimized to minimize the probability of arcing occurring to the emitter. The proposed cathode designs will be evaluated until two designs are chosen that offer promising results in mTorr oxygen pressure environments. The performance of these cathodes will be fully characterized before being integrated with a propulsion system.

\section{Acknowledgments}

This research is being funded by Dr. Len Caveny at BMDO and monitored by Dr. Jay Polk at the Jet Propulsion Laboratory (JPL). The authors would like to thank them for their support and Mission Research Inc. for giving the University of Michigan access to MAGIC. The expert advice received on field emitter technology was also greatly appreciated from several individuals. These people include Stella Pang, Capp Spindt, and Mohammad Rakhshandehroo.

\section{References}

Djuba, B. C. and N. N. Chubun (1991) "Emission Properties of Spindt-Type Cold Cathodes with Different Emission Cone Material," IEEE Trans. Electron Devices, 38(10):2314.

Khayms, V. and M. Martinez-Sanchez (1996) "Design of A Mineaturized Hall Thruster for Microsatellites," AIAA 96-3291, July 1996.

Lide, D.R. (ed) (1992), Handbook of Chemistry and Physics, 73rd. ed. 1992-1993, CRC Press Inc.

Mackie, W. A., J. L. Morrissey, C. H. Hindrichs, and P.R. Davis (1992) "Field Emission from Hafnium Carbide," Journal of Vacuum Science and Technology A 10(4), July/Aug 1992.

Mackie, W. A., R. L. Hartman, and P. R. Davis (1993) "High Current Density Field Emission from Transition Metal Carbides," Applied Surface Science, 67:29-35.

Mackie, W. A., R.L. Hartman, M. A. Anderson, and P. Davis (1994) "Transition Metal Carbides for use as Field Emission Cathodes," Journal of Vacuum Science and Technology B, 12(2)722:726, Mar/Apr 1994

Mackie, W. A., T. Xie, and P. R. Davis (1995) "Field Emission from Carbide Film Cathodes," Journal of Vacuum Science and Technology, B 13(6), Nov/Dec 1995.

Manzella, D., S. Oleson, J. Sankovic, T. Haag, A. Semenkin, and V. Kim (1996) "Evaluation of Low Power Hall Thruster Propulsion," AIAA-96-2736, July 1996.

Mousa, M. S., P. R. Schwoebel, I. Brodie, and C. A. Spindt 1993 "Observations of Work Function Changes in Field-Emitter Arrays," Applied Surface Science, 67:56-58

Price, D. L., B. R. Cooper, and J. M. Mills (1993) "Effect of Carbon Vacancies on Carbide Workfunctions," The American Physical Society, Physical Review B, 48(20), Nov.1993.

Schwoebel, P. R., C. A. Spindt, and I. Brodie (1995) "Electron Emission Enhancement by Overcoating Molybdenum Field Emitter Arrays with Titanium, Zirconium, and Hafnium," Journal of Vacuum Science and Technol. B 13(2):338, Mar/Apr 1995. 